\title{
Genome-wide linkage analyses of hereditary prostate cancer families with colon cancer provide further evidence for a susceptibility locus on 15q11-q14
}

\author{
Liesel M FitzGerald ${ }^{1,6}$, Shannon K McDonnell ${ }^{2,6}$, Erin E Carlson ${ }^{2}$, Wendy Langeberg${ }^{1}$, Laura M McIntosh ${ }^{1}$, \\ Kerry Deutsch ${ }^{3}$, Elaine A Ostrander ${ }^{4}$, Daniel J Schaid ${ }^{2}$ and Janet L Stanford ${ }^{\star, 1,5}$
}

The search for susceptibility loci in hereditary prostate cancer (HPC) is challenging because of locus and disease heterogeneity. One approach to reduce disease heterogeneity is to stratify families on the basis of the occurrence of multiple cancer types. This method may increase the power for detecting susceptibility loci, including those with pleiotropic effects. We have completed a genome-wide SNP linkage analysis of 96 HPC families, each of which has one or more first-degree relatives with colon cancer (CCa), and further analyzed the subset of families with two or more CCa cases $(n=27)$. When only a prostate cancer (PCa) phenotype was considered to be affected, we observed suggestive evidence for linkage (LOD $\geq 1.86$ ) at 15q14, 18q21 and $19 q 13$ in all families, and at $1 \mathrm{p} 32$ and $15 q 11-q 14$ in families with two or more CCa cases. When both the PCa and CCa phenotypes were considered affected, suggestive evidence for linkage was observed at 11q25, 15q14 and 18q21 in all families, and at 1q31, 11q14 and 15q11-14 in families with two or more CCa cases. The strongest linkage signal was identified at $15 q 14$ when both PCa and CCa phenotypes were considered to be affected in families with two or more CCa cases (recessive $H L O D=3.88$ ). These results provide further support for the presence of HPC susceptibility loci on chromosomes $11 q 14$, $15 q 11-q 14$ and $19 q 13$ and highlight loci at $1 q 31,11 q, 15 q 11-14$ and 18q21 as having possible pleiotropic effects. This study shows the benefit of using a comprehensive family cancer history to create more genetically homogenous subsets of HPC families for linkage analyses.

European Journal of Human Genetics (2010) 18, 1141-1147; doi:10.1038/ejhg.2010.49; published online 21 April 2010

Keywords: prostate cancer; colon cancer; linkage analysis; pleiotropic effects; hereditary

\section{INTRODUCTION}

Family-based genome-wide linkage studies have highlighted over two dozen putative loci for hereditary prostate cancer (HPC) with significant or suggestive signals; however, because of the joint contribution of locus and disease heterogeneity, few have been confirmed and most have not yielded clear evidence of specific genes or causative variants (reviewed in Ostrander et al ${ }^{1}$ and Schaid ${ }^{2}$ ). To overcome some of these challenges and create more homogenous subsets of pedigrees, linkage studies have focused on refining the phenotypic definition of prostate cancer (PCa). ${ }^{3,4}$

One approach to overcome heterogeneity in a complex disease such as HPC is to stratify families by the presence of other primary cancers. This method is supported by evidence that some inherited susceptibility genes have a pleiotropic effect. ${ }^{5}$ Although a cancer syndrome that includes PCa has not been identified, there is evidence that relatives of PCa probands are at increased risk for other primary cancers such as colon. ${ }^{6}$ Analysis of a subset of HPC families in which there is an occurrence of an additional cancer type offers the advantage of reducing locus heterogeneity, thereby improving power for finding susceptibility loci.
Stratifying families on the basis of the presence of multiple cancers has been used successfully to map loci and then characterize the $B R C A 1$ and BRCA2 genes for breast cancer. ${ }^{7-10}$ Although specific loci have not yet been identified, PCa linkage studies of families with multiple cancers have also suggested regions that may contain HPC susceptibility genes. The putative 1p36 HPC susceptibility region was initially mapped using families with first-degree relatives diagnosed with primary brain cancer, ${ }^{11}$ with subsequent evidence for this locus reported in other studies. ${ }^{12,13}$ Linkage analyses of HPC families stratified by the presence of kidney cancer ${ }^{14}$ or pancreas cancer ${ }^{15}$ in first-degree relatives of PCa probands have also suggested susceptibility loci, two of which, 7 p21 and 16q23, have also been identified in other HPC linkage analyses. ${ }^{4,16,17}$ Evidence for a shared genetic etiology of $\mathrm{PCa}$ and colon cancer (CCa) has emerged from studies showing an excess risk of CCa in families ascertained for PCa, and vice versa. ${ }^{6,18,19}$ In addition, several susceptibility regions, including 3 p14, 8q24 and 15q13-q14, have been suggested in both $\mathrm{PCa}^{16,20-24}$ and $\mathrm{CCa}^{20,25-27}$ studies, providing support for a potential shared genetic disposition for developing these two cancers.

\footnotetext{
${ }^{1}$ Division of Public Health Sciences, Fred Hutchinson Cancer Research Center, Seattle, WA, USA; ${ }^{2}$ Division of Biostatistics, Mayo Clinic, Rochester, MN, USA; ${ }^{3}$ Institute for Systems Biology, Seattle, WA, USA; ${ }^{4}$ Cancer Genetics Branch, NHGRI, National Institutes of Health, Bethesda, MD, USA; ${ }^{5}$ epartment of Epidemiology, School of Public Health, University of Washington, Seattle, WA, USA

*Correspondence: Dr JL Stanford, Division of Public Health Sciences, Fred Hutchinson Cancer Research Center, M4-B874, PO B0X 19024, Seattle, WA 98109-1024, USA. Tel: +1206 667 2715; Fax: +1206 667 2717; E-mail: jstanfor@fhcrc.org

6These authors contributed equally to this work.

Received 23 September 2009; revised 28 January 2010; accepted 10 March 2010; published online 21 April 2010
} 
On the basis of the evidence presented above, we have selected 96 HPC families with at least one first-degree relative of a PCa proband with $\mathrm{CCa}$ and performed genome-wide parametric and nonparametric linkage analyses.

\section{MATERIALS AND METHODS}

Hereditary prostate cancer families with colon cancer members

HPC families included in these analyses are participating in the Prostate Cancer Genetic Research Study (PROGRESS). ${ }^{24,28}$ Participants have completed a baseline and two follow-up surveys that queried personal and family cancer history. From these questions, we identified families that had one or more members with CCa. An individual was considered to have a diagnosis of CCa if (1) it was self-reported; (2) there was at least one first-degree relative report; and/or (3) there were at least two second-degree relative reports. A random sample of $27 \mathrm{CCa}$ cases was chosen for reporting validation. For the 11 individuals for whom a medical record or death certificate was obtained, $10 \mathrm{CCa}$ (91\%) reports were confirmed. In addition to the above 27 cases, death certificates were available for another 23 CCa cases, 12 of whom also had PCa. Of these, $100 \%$ of the 23 CCa reports were confirmed (five selfreports and 18 relative reports). Families were only considered eligible for this study if the individual(s) with CCa had a first-degree relative with PCa. Individuals with CCa were also considered to be affected if they had a firstdegree relative with CCa who, in turn, had a first-degree relative with PCa. All other CCa cases were considered unknown. Using the above criteria, $136 \mathrm{CCa}$ cases were identified in 99 PROGRESS families. In all, 57 of the CCa cases (41.9\%) also had PCa. A total of 98 CCa cases had genotyping data, of which 43 were inferred. Families were of Caucasian $(N=95)$, Hispanic $(N=1)$ or other $(N=3)$ ancestry. The analyses reported in this study included only the 96 Caucasian/Hispanic HPC families with one or more CCa cases as summarized in Table 1.

\section{Genotyping and quality control}

Genotyping and quality control analyses were performed as a part of a larger study described previously. ${ }^{24}$ In brief, samples were genotyped using the Illumina Linkage Panel IVb (5867 SNPs; Illumina, Inc., San Diego, CA, USA) and analyses were performed on a total of 4743 tagSNPs with a median minor allele frequency (MAF) of 0.40 (range $0.05-0.50$ ), a median call rate of $99 \%$ (range $97-100 \%$ ), a median intermarker distance over all chromosomes of $0.60 \mathrm{~cm}$ (range $0.001-5.97 \mathrm{~cm}$ ) and a median overall information content of $86 \%$ (range 63-90\%).

\section{Linkage analysis}

Both parametric and nonparametric allele-sharing linkage analyses were performed using Merlin software (http://www.sph.umich.edu/csg/abecasis/ Merlin/index.html). ${ }^{29}$ Parametric LOD scores were computed using an assumed PCa susceptibility allele frequency of 0.003 and 0.15 for autosomal dominant and recessive models, respectively, with a fixed phenocopy rate of $15 \%$. Penetrances of 0.001 for noncarriers and 1.0 for carriers of a putative risk allele were assumed. Parametric LOD scores allowed for linkage heterogeneity (HLOD) by estimating the fraction of linked pedigrees. Nonparametric LOD scores were calculated using the Kong and Cox exponential allele-sharing model score (KCLOD). ${ }^{30}$

Parametric LOD scores were based on an 'affecteds-only' analysis. For this approach, the genotypes of all pedigree members were used, but only affecteds were coded as such; unaffected members were coded as unknown phenotype. This strategy eliminates modeling the penetrance for unaffected subjects, who might not be thoroughly screened for disease. Furthermore, the absolute values of the assumed penetrances are not critical, only the ratio of penetrances (eg, as a genotype relative risk). However, including the genotypes of subjects with unaffected or unknown phenotypes does increase the linkage information content by increasing the identity-by-descent information among affected subjects.

Our analyses considered PCa and CCa in two different ways: (1) only individuals with PCa were coded as affected (subjects with CCa were coded as unknown); and (2) individuals with either PCa or CCa were coded as affected. This allowed us to evaluate the contribution of CCa to the linkage results.

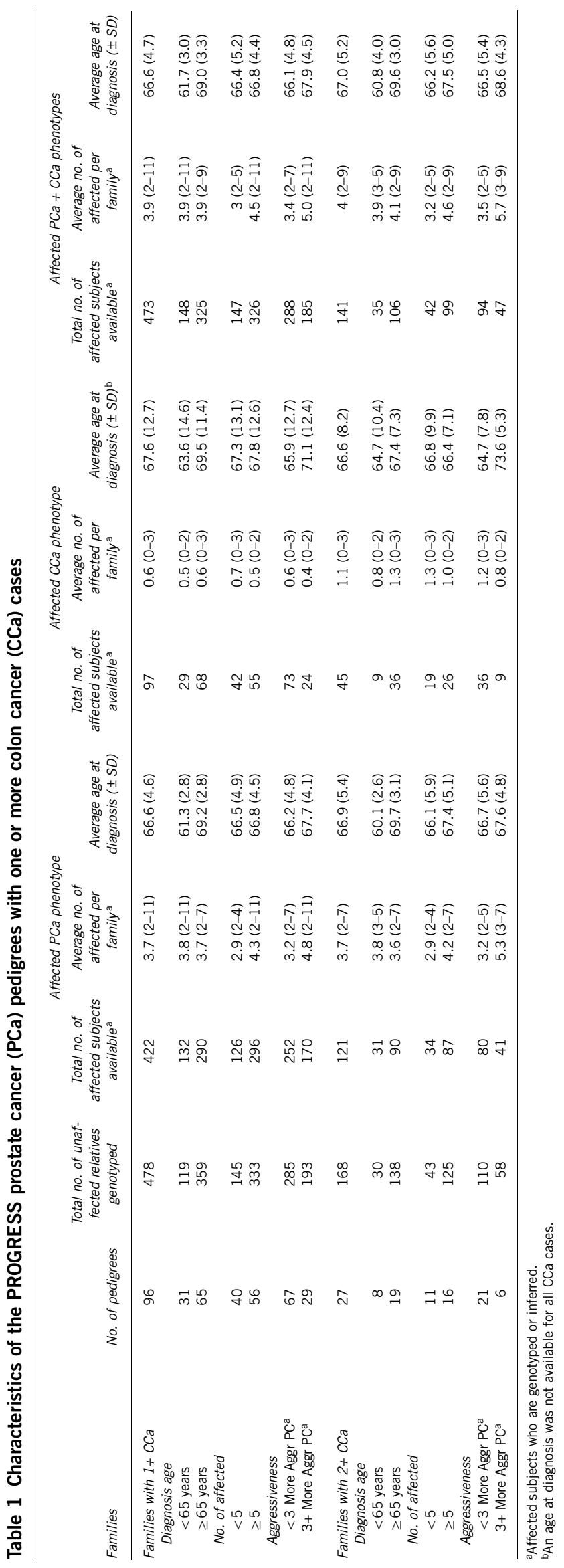


The parametric linkage models were the same for both analyses (in terms of assumed allele frequencies and penetrances).

Although Merlin software can analyze SNPs that are in linkage disequilibrium (LD) by treating them as multiallelic markers and using the expectation-maximization algorithm, ${ }^{31}$ this option requires an excessive amount of memory and analytical time for large pedigrees. We thus eliminated much of the LD by selecting 4743 tagSNPs using LDselect ${ }^{32}$ with a low $r^{2}$ threshold $\left(r^{2} \leq 0.10\right)$; tags for each bin were chosen considering Illumina QC measures, MAF and SNP call rate. Marker allele frequencies were estimated across the pool of all subjects, ignoring genetic relationships.

Pedigrees were stratified as follows: (1) families with one or more colon cancer cases ( 96 families); and (2) families with two or more colon cancer cases (27 families). One HPC-colon cancer pedigree had to be split into two subpedigrees to 'fit' the data into the memory limits of Merlin software.

\section{RESULTS}

The findings presented in this study are based on 96 HPC-colon cancer pedigrees in which 900 individuals were genotyped $(n=835)$ or genotype information was reconstructed from relatives $(n=65)$. These individuals included 422 PCa cases, 67 male CCa cases (46 of whom also had a diagnosis of PCa) and 30 female CCa cases (Table 1). A total of 27 of the 96 pedigrees had at least two affected subjects with CCa.

Figure 1 presents the LOD scores for the HPC-colon cancer families analyzed for the PCa-only phenotype. LOD scores of at least 1.86 were considered suggestive for linkage, ${ }^{33}$ and these are summarized in Table 2. When considering families with one or more CCa cases, suggestive evidence for linkage was observed on chromosomes 15 and 19 under a recessive model $(15 \mathrm{q} 14 \mathrm{HLOD}=1.90$ at $33 \mathrm{Mb}$ and $19 \mathrm{q} 13.33 \mathrm{HLOD}=1.96$ at $56 \mathrm{Mb}$ ) and on $18 \mathrm{q} 21.2$ with a dominant HLOD of 1.87 at $49 \mathrm{Mb}$. Considering the 27 families with two or more CCa cases, the most striking linkage signal was detected on chromosome $15 \mathrm{q} 14$, with a recessive HLOD of 3.47 at $33 \mathrm{Mb}$. Further suggestive evidence for linkage on chromosome 15 was present at $15 \mathrm{q} 11-\mathrm{q} 14$ (KCLOD $=2.85$ on $15 \mathrm{q} 11.2$ at $22 \mathrm{Mb}$ and dominant $\mathrm{HLOD}=2.22$ on $15 \mathrm{q} 13.3-\mathrm{q} 14$ at $31 \mathrm{Mb}$ ). There was also suggestive evidence for linkage on chromosome $1 \mathrm{p} 32.3$, with a recessive HLOD of 2.45 at $54 \mathrm{Mb}$.

Figure 2 presents the LOD scores for HPC-colon cancer families analyzed for PCa and CCa phenotypes. Suggestive LOD scores of at least 1.86 are presented in Table 2. Considering all families with one or more CCa cases, suggestive evidence for linkage was observed at chromosome 11q25 (KCLOD=1.94 at $134 \mathrm{Mb}), 15 \mathrm{q} 14$ (recessive $\mathrm{HLOD}=2.13$ at $33 \mathrm{Mb}$ ) and $18 \mathrm{q} 21.2$ (dominant $\mathrm{HLOD}=2.82$ at $\sim 50 \mathrm{Mb}$ ). In the subset of families with two or more CCa cases, suggestive evidence for linkage under a recessive model was observed on chromosome 1q31.3 (HLOD=2.19 at $\sim 197 \mathrm{Mb}$ ) and $11 \mathrm{q} 14.1$ $(\mathrm{HLOD}=2.35 \mathrm{at} 81 \mathrm{Mb})$. Again, the most striking evidence for linkage
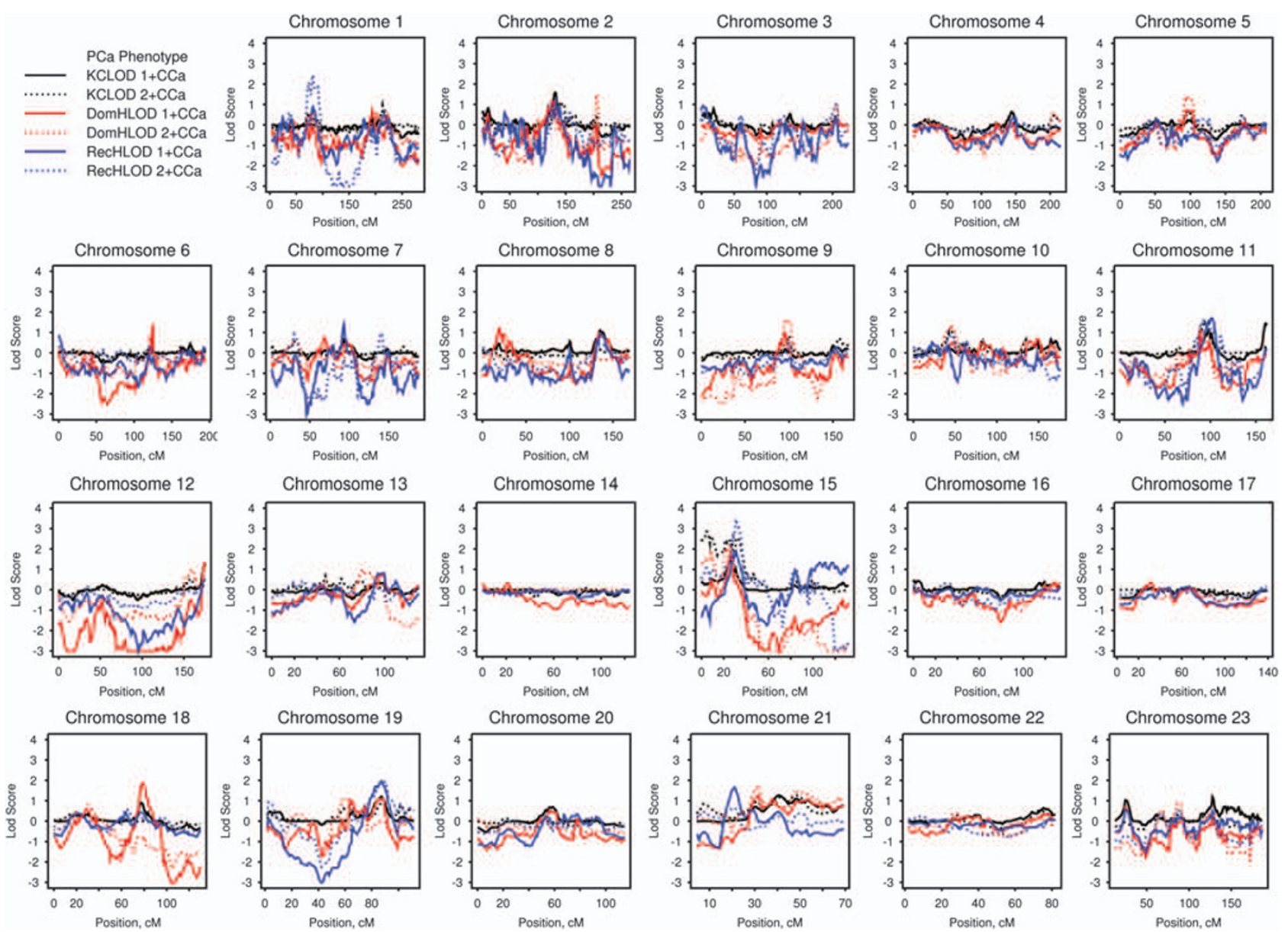

Figure 1 Genome-wide linkage results for the prostate cancer phenotype in hereditary prostate cancer-colon cancer families. Solid lines denote analyses of all 96 families and dashed lines denote analyses of the 27 hereditary prostate cancer families with two or more colon cancer cases. DomHLOD (red) and RecHLOD (blue) are the dominant and recessive heterogeneity LOD scores under an affecteds-only model. KCLOD (black) is the Kong and Cox exponential allele-sharing model LOD score. 
Table 2 Suggestive LOD scores (LOD $\geq 1.86)$ for prostate cancer and for prostate and colon cancer (CCa) phenotypes

\begin{tabular}{|c|c|c|c|c|c|c|c|c|c|}
\hline \multirow[b]{3}{*}{ Chromosome } & \multirow[b]{3}{*}{$c M$} & \multirow[b]{3}{*}{ Left flanking SNP (bp) } & \multirow[b]{3}{*}{ Right flanking SNP (bp) } & \multicolumn{6}{|c|}{ Phenotype } \\
\hline & & & & \multicolumn{3}{|c|}{ Prostate cancer } & \multicolumn{3}{|c|}{ Prostate and colon cancer } \\
\hline & & & & $K \& C^{\mathrm{a}} \angle O D$ & $\begin{array}{c}\text { Dominant } \\
H L O D^{\mathrm{b}}\end{array}$ & $\begin{array}{c}\text { Recessive } \\
H L O D^{\mathrm{b}}\end{array}$ & $K \& C^{\text {a }} L O D$ & $\begin{array}{c}\text { Dominant } \\
H L O D^{\mathrm{b}}\end{array}$ & $\begin{array}{c}\text { Recessive } \\
H L O D^{\mathrm{b}}\end{array}$ \\
\hline \multicolumn{10}{|c|}{ Families with $1+\mathrm{CCa}(n=96)$} \\
\hline $11 q 25$ & 162.07 & rs1824832 (134313007) & rs4430531 (134447835) & 1.46 & -0.06 & 0.22 & 1.94 & -0.04 & 0.43 \\
\hline $15 q 13-14$ & 25.7 & rs343913 (31133168) & rs2033610 (31542203) & 1.46 & 1.76 & 0.75 & 0.99 & 0.40 & 1.02 \\
\hline $18 q 21$ & 79.39 & rs1145315 (49942953) & rs1942569 (50997722) & 0.7 & 1.84 & 0.39 & 1.08 & 2.82 & 0.59 \\
\hline $19 q 13$ & 87.21 & rs897783 (56722974) & rs1993104 (56932060) & 1.21 & 1.10 & 1.96 & 1.14 & 0.55 & 0.79 \\
\hline \multicolumn{10}{|c|}{ Families with $2+\mathrm{CCa}(n=27)$} \\
\hline $1 p 32$ & 80.95 & rs731828 (54140841) & rs1537323 (54255111) & 0.53 & 0.53 & 2.45 & 0.75 & -0.23 & -0.47 \\
\hline $15 q 14$ & 31.04 & rs732165 (33141489) & rs1989223 (33914397) & 2.45 & 0.77 & 3.47 & 2.29 & 0.41 & 3.88 \\
\hline
\end{tabular}

aK\&C, Kong and Cox exponential allele-sharing model.

bAffecteds-only model (see text).

was observed on chromosome $15 \mathrm{q} 14$ with a recessive HLOD of 3.88 at $33 \mathrm{Mb}$; however, the KCLOD decreased slightly at this locus compared with that observed for the PCa-only phenotype (KCLOD of 2.29 and 2.45 at $33 \mathrm{Mb}$, respectively). An additional suggestive linkage peak was observed at $15 \mathrm{q} 11.2$ with a KCLOD of 2.14 at $22 \mathrm{Mb}$, although evidence for linkage again decreased slightly when the CCa phenotype was considered as affected.

Because CCa, similar to $\mathrm{PCa}$, is a relatively common cancer with both hereditary and sporadic forms, we analyzed whether the stratification of families by the number of CCa cases affected our strongest linkage signal at 15q11-14. We reanalyzed the data according to the number of CCa cases: one case $v s$ two or more cases per family. Each stratum was analyzed for the PCa-only phenotype and then for the $\mathrm{PCa}$ and $\mathrm{CCa}$ phenotypes. Figure 3 shows that under both a nonparametric and recessive model, HPC families with only one CCa case provided no evidence for linkage to 15q11-14, whereas families with two or more CCa cases provided all evidence for linkage to this region.

\section{DISCUSSION}

To create a comparatively more homogeneous subset of HPC pedigrees, we analyzed data from 96 HPC families with at least one CCa case among first-degree relatives of a PCa proband. Families were initially analyzed using a PCa-only phenotype. To search for loci with possible pleiotropic effects, analyses were repeated considering both PCa and CCa phenotypes as affected. Several suggestive linkage signals were identified, with the most striking evidence observed at 15q14 in families with two or more $\mathrm{CCa}$ cases and both PCa and CCa phenotypes considered affected (recessive $\mathrm{HLOD}=3.88$ ).

Several of the linkage peaks observed in this study have been noted in previous PCa linkage studies, including $11 \mathrm{q} 14,{ }^{34,35} 19 \mathrm{q} 13^{36,37}$ and, of particular interest, 15q11-14. The International Consortium for Prostate Cancer Genetics (ICPCG) presented suggestive evidence for linkage to $15 \mathrm{q} 11$ (recessive $\mathrm{LOD}=2.10$ ) in a microsatellite analysis of 1233 HPC families, including 254 PROGRESS families. ${ }^{38}$ A study of
230 multiplex PCa sibships from the United States ${ }^{16}$ found a $Z$-score of 2.77 at $15 \mathrm{q} 13.3$ and when investigating 139 families from Germany, ${ }^{23}$ a $Z$-score of 2.23 was observed at 15 q13.1. In 2004, a study of 44 Japanese PCa families highlighted a linkage peak at $15 \mathrm{q} 14$ $\left(Z_{\mathrm{lr}}=1.75\right),{ }^{12}$ and, most recently, $15 \mathrm{q} 13-\mathrm{q} 14$ was highlighted in a dense SNP genome-wide scan of 289 Caucasian HPC families $(\mathrm{HLOD}=1.99)$, which included the 96 pedigrees presented in this study. ${ }^{24}$ Interestingly, the strongest evidence for linkage at this region was in a subset analysis of 128 families with younger ages at diagnosis $(\mathrm{KCLOD}=2.82),{ }^{24}$ eight of which overlap with the 27 HPC-colon cancer families that provided the strongest evidence for linkage in the current analyses (recessive HLOD=3.88). Although stratifying by age at diagnosis seemed to create some homogeneity in the original analyses of the PROGRESS data set, ${ }^{24}$ the method of selecting families on the basis of the presence of another primary cancer, in this case $\mathrm{CCa}$, clearly created greater homogeneity among the families, especially in families with two or more CCa cases. Interestingly, families contributing evidence for linkage to one locus rarely provided evidence for linkage to any of the other loci, further suggesting that this study achieved both inter- and intra-familial heterogeneity.

On the basis of several lines of evidence, our a priori hypothesis was that a shared genetic etiology exists for PCa and CCa. First, an excess risk of $\mathrm{CCa}$ has been reported in families ascertained for $\mathrm{PCa}^{6,18}$ and, conversely, an excess of PCa $(\mathrm{RR}=1.3,95 \%$ CI 1.1-1.4) has been observed in families ascertained for CCa. ${ }^{19}$ Second, a study by Ozden et $a l^{39}$ found a $10.7 \%$ 10-year cumulative incidence of PCa in men with a history of CCa compared with $3.8 \%$ in men without CCa. Third, mutations in two of the mismatch repair genes (MLH1 and $M S H 3)$, which are associated with predisposition to hereditary nonpolyposis colon cancer (HNPCC), have been associated with an increased relative risk of $\mathrm{PCa} .{ }^{40,41}$ Of particular interest to the work reported here are earlier studies that have highlighted several common susceptibility regions for both PCa and CCa, including $3 \mathrm{p} 14.2^{21,26}$ 15q13-q14 $4^{16,23-25}$ and $18 \mathrm{q} 21 .^{24,42}$ Finally, a PCa linkage study highlighted the $8 \mathrm{q} 24$ locus $^{22}$ that has since been shown to harbor a SNP, 

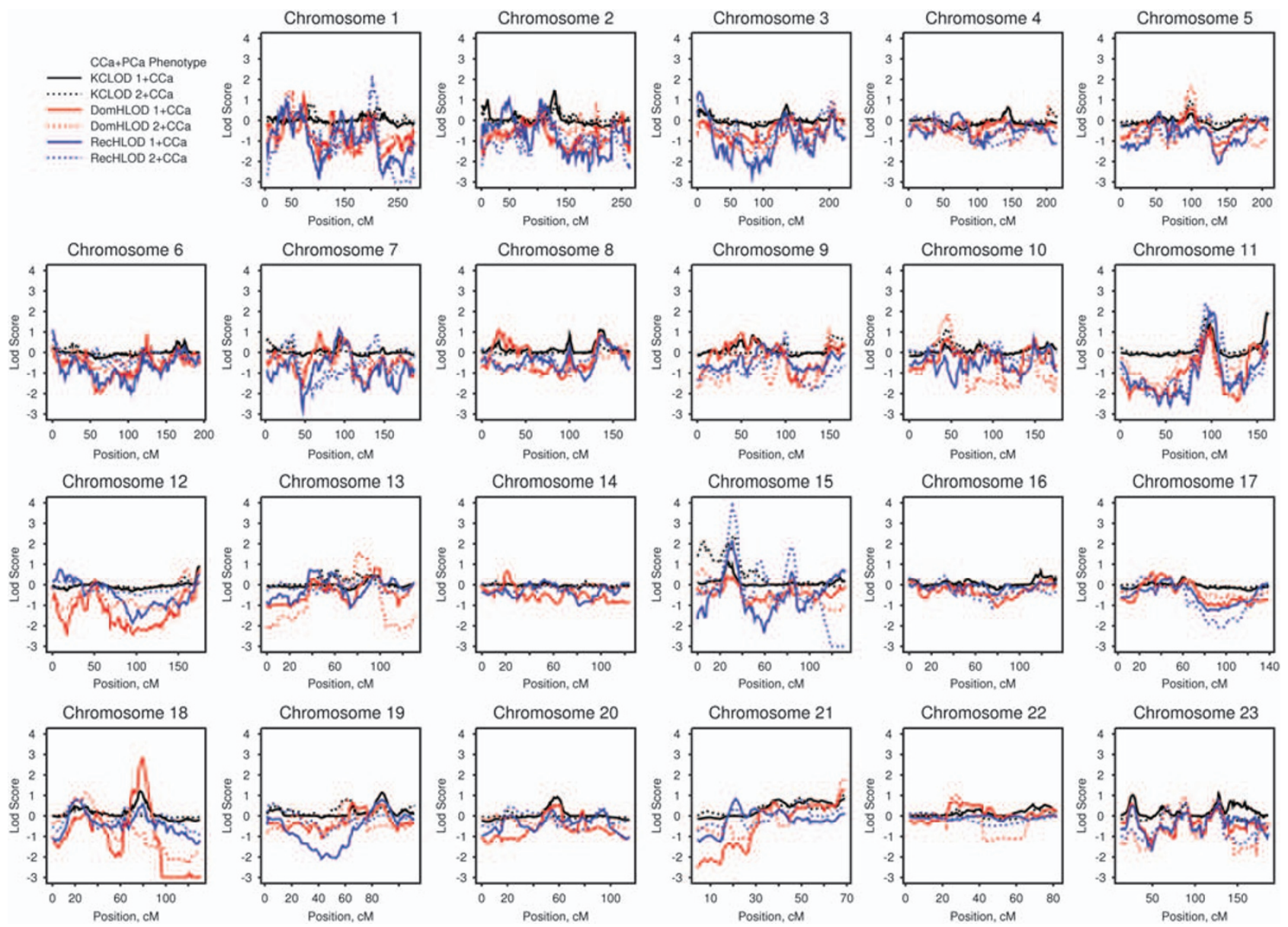

Figure 2 Genome-wide linkage results for prostate and colon cancer phenotypes in hereditary prostate cancer-colon cancer families. Solid lines denote analyses of all 96 families and dashed lines denote analyses of the 27 hereditary prostate cancer families with two or more colon cancer cases. DomHLOD (red) and RecHLOD (blue) are the dominant and recessive heterogeneity LOD scores under an affecteds-only model. KCLOD (black) is the Kong and Cox exponential allele-sharing model LOD score.

rs6983267, which is associated with increased relative risks of both PCa and CCa. ${ }^{20,43}$

The fact that several of the regions observed in this study have been previously observed in PCa or CCa studies, or both, is noteworthy. The 15q11-q14 region was first suggested as a CCa susceptibility locus based on linkage analyses of Ashkenazi Jewish pedigrees. ${ }^{27}$ More recently, a linkage scan of colon neoplasia families also highlighted 15q14-q22..$^{25}$ Jaeger et al ${ }^{44}$ have restricted the location of the CCa susceptibility locus to a region spanning approximately $1 \mathrm{Mb}$ (30.7-31.4 Mb), which overlaps with the larger region of linkage presented in this study $(22.3-33.9 \mathrm{Mb})$. Then, in a large association study of CCa in cases from the United Kingdom a SNP from this same region was shown to increase risk in cases with a family history or an early onset of disease ( $r$ 45779584; $\left.P=4.44 \times 10^{-14}\right){ }^{44}$ This is particularly interesting, as $15 \mathrm{q} 13-14$ has been highlighted previously in PROGRESS families with a younger mean age at diagnosis. ${ }^{24}$

The 15q11-14 peak observed in this study encompasses an interesting candidate gene, FMN1, which is highly expressed in the prostate. The formin (FMN) family of proteins governs cytoskeletal organization in cells and is known to have an essential role in cell division and polarity. ${ }^{45,46}$ Although a link between FMN1 and cancer has not yet been described, the peak signals for the above-mentioned $\mathrm{US}^{16}$ and German ${ }^{23} \mathrm{PCa}$ linkage analyses also fall within the transcript region of the FMN1 gene. Intriguingly, the colorectal susceptibility locus identified in the study by Jaeger et $a^{44}$ also spans the FMN1 gene (30.7-31.4 Mb).

Three other regions highlighted in this study, 11q14, 11q25 and 18q21, have also been observed in PCa and/or CCa studies. The 11q14 region has been noted not only in PCa linkage studies ${ }^{34,35}$ but also in a genome-wide linkage analysis of 18 Swedish CCa families. ${ }^{47}$ Although 11q25 has been highlighted in previous PCa linkage studies, ${ }^{23,24}$ recent evidence suggests that $11 \mathrm{q} 25$ may contain a locus that is involved in multiple malignancies. Cui et al ${ }^{48}$ have shown that the $11 \mathrm{q} 25$ tumorsuppressor gene, $O P C M L$, is downregulated by promoter methylation in several tumor cell lines, including prostate, colon and breast. Chromosome 18q21 has also been suggested as being both a $\mathrm{PCa}$ and CCa susceptibility region through $\mathrm{LOH}$ studies, ${ }^{49,50}$ a previous linkage analysis of PROGRESS families with a comparatively more clinically aggressive PCa phenotype and an older mean age at diagnosis, ${ }^{24}$ and, finally, a GWAS of familial CCa cases and controls (rs4939827; $\left.P=1.0 \times 10^{-12}\right) .51$

There were a number of linkage peaks noted in this study that were either stronger or only present when analyzing a specific phenotype. For example, peaks at $19 \mathrm{q} 13$ and $1 \mathrm{p} 32$ were stronger or only present, respectively, when analyzing the PCa phenotype, whereas peaks at $11 \mathrm{q} 14$ and $1 \mathrm{q} 31$ were stronger or only present, respectively, when 


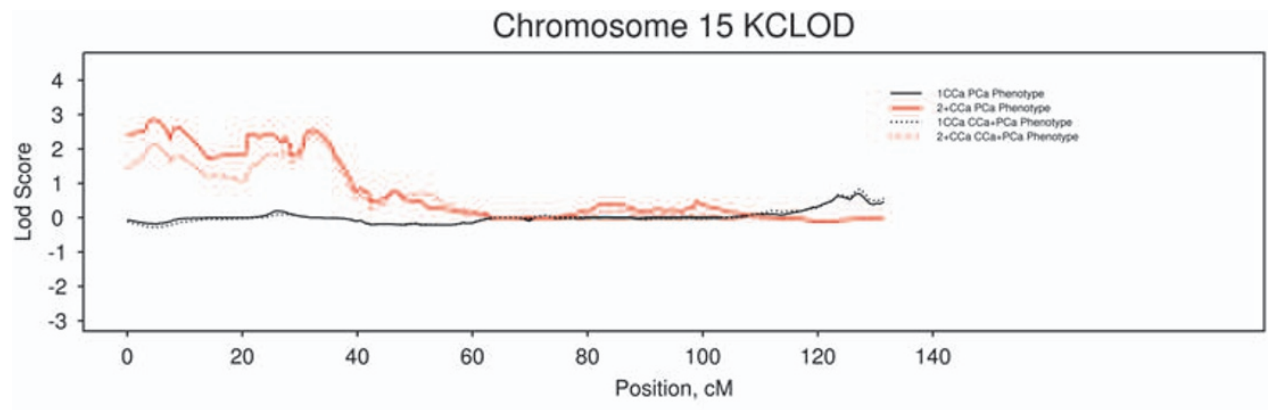

Chromosome 15 Recessive

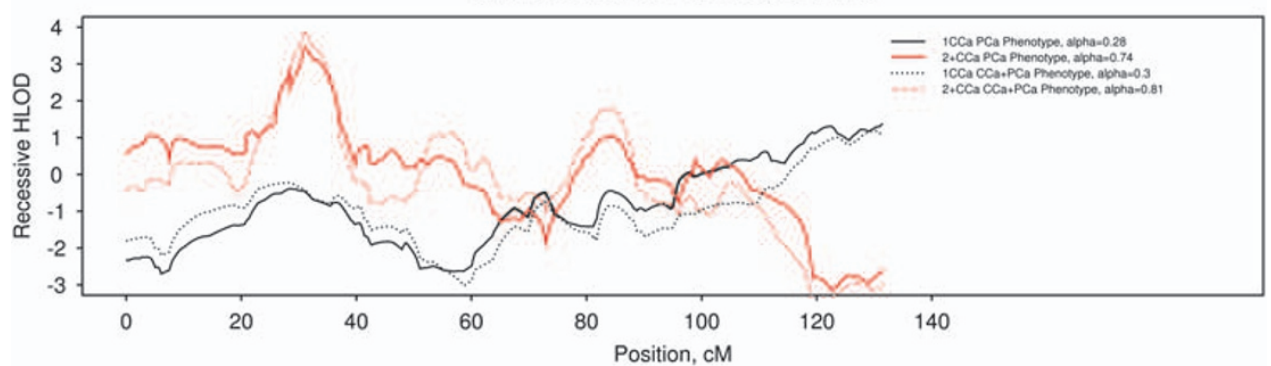

Figure 3 Linkage results for chromosome 15 under a nonparametric (top panel) and recessive (lower panel) model of a prostate cancer phenotype (solid lines) and both prostate and colon cancer phenotypes (dashed lines). Analyses of hereditary prostate cancer families with one colon case are denoted with a black line and analyses of hereditary prostate cancer families with two or more colon cancer cases are denoted with a red line.

analyzing both the PCa and CCa phenotypes. Although none of these regions reached genome-wide statistical significance, these results suggest that specific phenotype analyses may reveal susceptibility loci that have either disease-specific effects (1p32 and 19q13) or potential pleiotropic effects (1q31 and 11q14).

One limitation of this study is that not all $\mathrm{CCa}$ cases were confirmed by medical records or death certificates. Such documentation was obtained for only $34(25.6 \%)$ of the CCa cases, but these records confirmed $97 \%$ of the CCa diagnoses in this subset. Previous studies have found that $17-100 \%$ of self-reported $\mathrm{CCa}^{52,53}$ and $48-93 \%$ of first-degree relative-reported CCa were confirmed by medical records. ${ }^{54,55}$ Therefore, there may be individuals with CCa who were missed or, more rarely, individuals with an incorrect assignment of CCa. The first scenario should not contribute to false findings of linkage, as all our analyses relied on phenotype data only from affected individuals.

To our knowledge, in only rare instances does PCa metastasize to the colon, or vice versa; however, two additional issues that need to be considered when studying these two cancers are the potential effects of screening or early detection and treatment. Once a diagnosis of either PCa or CCa is made, the patient may be exposed to greater medical attention, increasing the likelihood of screening for additional cancer types. The diagnosis of the first primary cancer could also influence the patient's lifestyle choices and positive changes (eg, cessation of smoking, weight loss, increase in exercise), which may alter the risk of a subsequent cancer. Treatment for the first primary cancer may also affect the risk for subsequent cancers. A recent study found that PCa patients treated with external beam radiotherapy had an increased risk of CCa 5-9 years after treatment; ${ }^{56}$ however, previous studies have found discordant results regarding the occurrence of $\mathrm{CCa}$ and $\mathrm{PCa}$ and the subsequent risk of other cancers (reviewed in Bostrom and Solowa ${ }^{57}$ ). Of the 57 men in this study with earlier diagnoses of both primary $\mathrm{PCa}$ and primary $\mathrm{CCa}$, we have information regarding the temporal relationship between these diagnoses for 46 men. Of these, two men (4\%) were diagnosed with both cancers within the same year and five $(11 \%)$ men initially diagnosed with PCa and treated with primary radiation therapy were subsequently diagnosed with CCa 5-9 years after radiation treatment.

In summary, to create a more homogeneous subset of HPC families, a genome-wide linkage analysis of 96 families with at least one first-degree relative of a PCa proband with CCa was performed. This study has provided further support for the presence of HPC susceptibility regions on chromosomes 11q14, 15q11-q14, 18q21 and $19 \mathrm{q} 13$, with the most striking evidence for a region on $15 \mathrm{q} 14$. Thus, these results support both previously suggested HPC regions, as well as novel candidate HPC regions with potential pleiotropic effects. In fact, four well-recognized CCa susceptibility regions, 11q14, 11q25, 15q13-q14 and 18q21, were suggested as candidate PCa susceptibility regions in this study. Future work will focus on refining the interval of linkage at 15q11-q14 in HPC-colon families, with the aim of identifying the underlying susceptibility locus (loci). Although the search for highly penetrant, rare PCa susceptibility loci has proven to be difficult, the combination of approaches used in this study is likely to elucidate unique regions containing genes that are responsible for the heritable form of this common and complex disease.

\section{ACKNOWLEDGEMENTS}

We thank all the men and women who are participating in the PROGRESS study for their time, effort and cooperation. We also thank the study staff for help with ongoing data collection and processing. This work was supported by grants RO1-CA080122 and P50-CA097186 from the National Cancer Institute, with additional support from the Fred Hutchinson Cancer Research Center. Genotyping services were provided by the Center for Inherited Disease Research at Johns Hopkins University (contract N01-HG-65403 from the National Institutes of Health). We acknowledge the Prostate Cancer Foundation and the Intramural Program of the National Human Genome Research Institute.

\section{CONFLICT OF INTEREST}

The authors declare no conflict of interest. 
1 Ostrander EA, Markianos K, Stanford JL: Finding prostate cancer susceptibility genes. Annu Rev Genomics Hum Genet 2004; 5: 151-175.

2 Schaid DJ: The complex genetic epidemiology of prostate cancer. Hum Mol Genet 2004; 13 (Spec No 1): R103-R121.

3 Chang $\mathrm{BL}$, Isaacs SD, Wiley KE et al: Genome-wide screen for prostate cancer susceptibility genes in men with clinically significant disease. Prostate 2005; 64: 356-361.

4 Stanford JL, McDonnell SK, Friedrichsen DM et al: Prostate cancer and genetic susceptibility: a genome scan incorporating disease aggressiveness. Prostate 2006; 66: 317-325

5 Fearon ER: Human cancer syndromes: clues to the origin and nature of cancer. Science 1997: 278: 1043-1050.

6 Damber L, Gronberg H, Damber JE: Familial prostate cancer and possible associated malignancies: nation-wide register cohort study in Sweden. Int J Cancer 1998; 78: 293-297.

7 Hall JM, Lee MK, Newman B et al: Linkage of early-onset familial breast cancer to chromosome 17q21. Science 1990; 250: 1684-1689.

8 Miki Y, Swensen J, Shattuck-Eidens D et al: A strong candidate for the breast and ovarian cancer susceptibility gene BRCA1. Science 1994: 266: 66-71.

9 Thorlacius S, Tryggvadottir L, Olafsdottir GH et al: Linkage to BRCA2 region in hereditary male breast cancer. Lancet 1995; 346: 544-545.

10 Wooster R, Bignell G, Lancaster J et al: Identification of the breast cancer susceptibility gene BRCA2. Nature 1995; 378: 789-792.

11 Gibbs M, Stanford JL, Mclndoe RA et al: Evidence for a rare prostate cancersusceptibility locus at chromosome 1p36. Am J Hum Genet 1999; 64: 776-787.

12 Matsui $\mathrm{H}$, Suzuki K, Ohtake $\mathrm{N}$ et al: Genomewide linkage analysis of familial prostate cancer in the Japanese population. J Hum Genet 2004; 49: 9-15.

13 Badzioch M, Eeles R, Leblanc $\mathrm{G}$ et al: Suggestive evidence for a site specific prostate cancer gene on chromosome 1p36. The CRC/BPG UK Familial Prostate Cancer Study Coordinators and Collaborators. The EU Biomed Collaborators. J Med Genet 2000; 37: 947-949.

14 Johanneson B, Deutsch K, McIntosh L et al: Suggestive genetic linkage to chromosome $11 \mathrm{p} 11.2-q 12.2$ in hereditary prostate cancer families with primary kidney cancer. Prostate 2007; 67: 732-742.

15 Pierce BL, Friedrichsen-Karyadi DM, McIntosh L et al: Genomic scan of 12 hereditary prostate cancer families having an occurrence of pancreas cancer. Prostate 2007; 67: 410-415

16 Suarez BK, Lin J, Burmester JK et al: A genome screen of multiplex sibships with prostate cancer. Am J Hum Genet 2000; 66: 933-944.

17 Witte JS, Suarez BK, Thiel B et al: Genome-wide scan of brothers: replication and fine mapping of prostate cancer susceptibility and aggressiveness loci. Prostate 2003; 57: 298-308

18 Albright LA, Schwab A, Camp NJ, Farnham JS, Thomas A: Population-based risk assessment for other cancers in relatives of hereditary prostate cancer (HPC) cases. Prostate 2005; 64: 347-355.

19 Enblad P, Adami HO, Glimelius B, Krusemo U, Pahlman L: The risk of subsequent primary malignant diseases after cancers of the colon and rectum. A nationwide cohort study. Cancer 1990; 65: 2091-2100.

20 Haiman CA, Le Marchand L, Yamamato J et al: A common genetic risk factor for colorectal and prostate cancer. Nat Genet 2007; 39: 954-956.

21 Larson GP, Ding Y, Cheng LS et al: Genetic linkage of prostate cancer risk to the chromosome 3 region bearing FHIT. Cancer Res 2005; 65: 805-814.

22 Amundadottir LT, Sulem P, Gudmundsson J et al: A common variant associated with prostate cancer in European and African populations. Nat Genet 2006; 38: 652-658.

23 Maier C, Herkommer K, Hoegel J, Vogel W, Paiss T: A genomewide linkage analysis for prostate cancer susceptibility genes in families from Germany. Eur J Hum Genet 2005; 13: 352-360

24 Stanford JL, FitzGerald LM, McDonnell SK et al: Dense genome-wide SNP linkage scan in 301 hereditary prostate cancer families identifies multiple regions with suggestive evidence for linkage. Hum Mol Genet 2009; 18: 1839-1848.

25 Daley D, Lewis S, Platzer $\mathrm{P}$ et al: Identification of susceptibility genes for cancer in a genome-wide scan: results from the colon neoplasia sibling study. Am J Hum Genet 2008; 82: 723-736

26 Ohta M, Inoue H, Cotticelli MG et al: The FHIT gene, spanning the chromosome 3 p14.2 fragile site and renal carcinoma-associated $t(3 ; 8)$ breakpoint, is abnormal in digestive tract cancers. Cell 1996; 84: 587-597.

27 Tomlinson I, Rahman N, Frayling I et al: Inherited susceptibility to colorectal adenomas and carcinomas: evidence for a new predisposition gene on 15q14-q22. Gastroenterology 1999; 116: 789-795.

28 Janer M, Friedrichsen DM, Stanford JL et al: Genomic scan of 254 hereditary prostate cancer families. Prostate 2003; 57: 309-319.
29 Abecasis GR, Cherny SS, Cookson WO, Cardon LR: Merlin-rapid analysis of dense genetic maps using sparse gene flow trees. Nat Genet 2002; 30: 97-101.

30 Kong A, Cox NJ: Allele-sharing models: LOD scores and accurate linkage tests. $\mathrm{Am}$ Hum Genet 1997; 61: 1179-1188.

31 Abecasis GR, Wigginton JE: Handling marker-marker linkage disequilibrium: pedigree analysis with clustered markers. Am J Hum Genet 2005; 77: 754-767.

32 Carlson CS, Eberle MA, Rieder MJ, Yi Q, Kruglyak L, Nickerson DA: Selecting a maximally informative set of single-nucleotide polymorphisms for association analyses using linkage disequilibrium. Am J Hum Genet 2004; 74: 106-120.

33 Lander E, Kruglyak L: Genetic dissection of complex traits: guidelines for interpreting and reporting linkage results. Nat Genet 1995; 11: 241-247.

34 Schaid DJ, McDonnell SK, Zarfas KE et al: Pooled genome linkage scan of aggressive prostate cancer: results from the International Consortium for Prostate Cancer Genetics. Hum Genet 2006; 120: 471-485.

35 Schleutker J, Baffoe-Bonnie AB, Gillanders E et al: Genome-wide scan for linkage in Finnish hereditary prostate cancer (HPC) families identifies novel susceptibility loci at 11q14 and 3p25-26. Prostate 2003; 57: 280-289.

36 Eeles RA, Kote-Jarai Z, Giles GG et al: Multiple newly identified loci associated with prostate cancer susceptibility. Nat Genet 2008; 40: 316-321.

37 Slager SL, Schaid DJ, Cunningham JM et al: Confirmation of linkage of prostate cancer aggressiveness with chromosome 19q. Am J Hum Genet 2003; 72: 759-762.

$38 \mathrm{Xu} \mathrm{J}$, Dimitrov L, Chang BL et al: A combined genomewide linkage scan of 1,233 families for prostate cancer-susceptibility genes conducted by the international consortium for prostate cancer genetics. Am J Hum Genet 2005; 77: 219-229.

39 Ozden N, Saruc M, Smith LM, Sanjeevi A, Roy HK: Increased cumulative incidence of prostate malignancies in colorectal cancer patients. Int J Gastrointest Cancer 2003; 34: 49-54.

40 Burmester JK, Suarez BK, Lin JH et al: Analysis of candidate genes for prostate cancer. Hum Hered 2004; 57: 172-178.

41 Hirata $\mathrm{H}$, Hinoda $\mathrm{Y}$, Kawamoto $\mathrm{K}$ et al: Mismatch repair gene MSH3 polymorphism is associated with the risk of sporadic prostate cancer. J Urol 2008; 179: 2020-2024.

42 Curtin K, Lin WY, George R et al: Meta association of colorectal cancer confirms risk alleles at 8q24 and 18q21. Cancer Epidemiol Biomarkers Prev 2009; 18: 616-621.

43 Tomlinson I, Webb E, Carvajal-Carmona $\mathrm{L}$ et al: A genome-wide association scan of tag SNPs identifies a susceptibility variant for colorectal cancer at 8q24.21. Nat Genet 2007; 39: 984-988.

44 Jaeger $\mathrm{E}$, Webb $\mathrm{E}$, Howarth $\mathrm{K}$ et al: Common genetic variants at the CRAC1 (HMPS) locus on chromosome 15q13.3 influence colorectal cancer risk. Nat Genet 2008; 40: 26-28.

45 Wallar BJ, Alberts AS: The formins: active scaffolds that remodel the cytoskeleton. Trends Cell Biol 2003; 13: 435-446.

46 Zhou F, Leder P, Martin SS: Formin-1 protein associates with microtubules through a peptide domain encoded by exon-2. Exp Cell Res 2006; 312: 1119-1126.

47 Djureinovic T, Skoglund J, Vandrovcova J et al: A genome wide linkage analysis in Swedish families with hereditary non-familial adenomatous polyposis/non-hereditary non-polyposis colorectal cancer. Gut 2006; 55: 362-366.

48 Cui Y, Ying Y, van Hasselt A et al: OPCML is a broad tumor suppressor for multiple carcinomas and lymphomas with frequently epigenetic inactivation. PLOS ONE 2008; 3: e2990.

49 Frattini M, Balestra D, Suardi S et al: Different genetic features associated with colon and rectal carcinogenesis. Clin Cancer Res 2004; 10: 4015-4021.

50 Ueda T, Komiya A, Emi M et al: Allelic losses on 18q21 are associated with progression and metastasis in human prostate cancer. Genes Chromosomes Cancer 1997; 20: 140-147.

51 Broderick P, Carvajal-Carmona L, Pittman AM et al: A genome-wide association study shows that common alleles of SMAD7 influence colorectal cancer risk. Nat Genet 2007; 39: 1315-1317.

52 Navarro C, Chirlaque MD, Tormo MJ et al: Validity of self reported diagnoses of cancer in a major Spanish prospective cohort study. J Epidemiol Community Health 2006; 60: 593-599.

53 Sijmons RH, Boonstra AE, Reefhuis $\mathrm{J}$ et al: Accuracy of family history of cancer: clinical genetic implications. Eur J Hum Genet 2000; 8: 181-186.

54 Chang ET, Smedby KE, Hjalgrim H, Glimelius B, Adami HO: Reliability of self-reported family history of cancer in a large case-control study of lymphoma. J Natl Cancer Inst 2006; 98: 61-68.

55 Theis B, Boyd N, Lockwood G, Tritchler D: Accuracy of family cancer history in breast cancer patients. Eur J Cancer Prev 1994; 3: 321-327.

56 Rapiti E, Fioretta G, Verkooijen HM et al: Increased risk of colon cancer after external radiation therapy for prostate cancer. Int J Cancer 2008; 123: 1141-1145.

57 Bostrom PJ, Soloway MS: Secondary cancer after radiotherapy for prostate cancer: should we be more aware of the risk? Eur Urol 2007; 52: 973-982. 